\title{
Regulation of Vascular Growth and Regression by Matrix Metalloproteinases in the Rat Aorta Model of Angiogenesis
}

\author{
Wen-Hui Zhu, Xiaodu Guo, Sergio Villaschi, and Roberto Francesco Nicosia \\ VA Puget Sound Health Care System (WZ, RFN), and Department of Pathology (WZ, RFN), University of \\ Washington, Seattle, Washington; Department of Pathology (XG), National Cancer Institute, Bethesda, Maryland; \\ Dipartimento di Biopatologia (SV), Universita' di "Tor Vergata," Rome, Italy
}

\begin{abstract}
SUMMARY: Matrix metalloproteinases (MMPs) have been implicated in the formation of microvessels during angiogenesis, but their role in vascular regression is poorly understood. The rat aorta model of angiogenesis was used to study the function of MMPs at different stages of the angiogenic process. Gelatin zymography and Western analysis demonstrated production of MMP-2 and MMP-9 by aortic outgrowths in serum-free collagen gel culture. MMP-2 was found in both culture medium and collagen gel, whereas MMP-9 was predominantly associated with the gel. MMP expression increased gradually during the angiogenic growth phase and stayed high when vessels regressed and collagen lysed around the aortic rings. The MMP inhibitors, batimastat and marimastat, blocked formation of microvessels when added to the culture medium at the beginning of the experiment. They, however, stabilized the microvessels and prevented vascular regression after the angiogenic growth phase. This effect was observed also under conditions of angiogenic stimulation by basic fibroblast growth factor. MMP inhibitormediated stabilization of microvessels was associated with inhibition of collagen lysis and accumulation of collagen fibrils in the subendothelial space. This study demonstrates that MMPs promote microvessel formation during the early stages of angiogenesis, but also contribute to the reabsorption of the neovasculature in the later stages of this process. The time-dependent divergent effects of MMPs on microvessel growth and survival may influence the in vivo activity of MMP inhibitors used to treat angiogenesis-dependent disorders. (Lab Invest 2000, 80:545-555).
\end{abstract}

$A$ ngiogenesis, the formation of new blood vessels from pre-existing vessels, plays a fundamental role in embryonal development and physiologic/reactive processes, such as the female reproductive cycle and wound healing. New vessels contribute also to the progression of cancer, rheumatoid arthritis, diabetic retinopathy, and complicated atherosclerosis (Folkman, 1995; Folkman and Klagsbrun, 1987).

One of the initial steps in the angiogenic process is the degradation of the subendothelial basement membrane and surrounding extracellular matrix (ECM). Following matrix breakdown, endothelial cells migrate and proliferate to form new capillary tubes (Ausprunk and Folkman, 1977; Moscatelli and Rifkin, 1988). This process is mediated by ECM-degrading proteolytic enzymes, including matrix metalloproteinases (MMPs), which are secreted by endothelial cells in response to angiogenic stimuli (Cornelius et al, 1995; Ray and Stetler-Stevenson, 1994; Unemori et al, 1992).

The MMPs are a family of zinc-dependent endopeptidases, which are secreted as zymogens and activated

Received December 3, 1999

Supported by NIH HL52585

Address reprint requests to: Dr. R. F. Nicosia, Pathology and Laboratory Medicine (S-113-Lab), VA Puget Sound Health Care System, 1660 S. Columbian Way, Seattle, Washington 98108. Fax: (206) 764-2001; E-mail:Roberto.Nicosia@med.va.gov extracellularly by proteolytic cleavage. MMPs are classified into interstitial collagenases (substrate: interstitial collagen), stromelysins (substrates: laminin, fibronectin), gelatinases (substrate: type IV collagen), and membrane-type MMPs (MT-MMPs) (Ray and StetlerStevenson, 1994). MT-MMPs are a family of cell surfaceassociated enzymes that activate MMPs by proteolytic mechanisms. There is some functional overlap among the groups, because gelatinases such as MMP-2 can digest fibrillar collagen while stromelysins can digest type IV collagen. The function of the MMPs is regulated at multiple levels, including gene activation, transcription, mRNA stability, translation, secretion, binding to ECM components, and proenzyme activation and inactivation by tissue inhibitors of metalloproteinases (TIMPs) (Ray and Stetler-Stevenson, 1994).

In vitro studies have demonstrated that MMPs are involved in the formation of blood vessels. Microvascular endothelial cells cultured on Matrigel or in interstitial collagen gels penetrate the surrounding matrix and reorganize into tube-like structures. These morphogenetic changes are accompanied by endothelial secretion of proteolytic enzymes including MMPs (Haas et al, 1998; Schnaper et al, 1993). Synthetic MMP inhibitors and TIMPs block endothelial penetration of the ECM and capillary tube formation (Montesano et al, 1983; Ray and Stetler-Stevenson, 1994; Schnaper et al, 1993). Expression of MMPs by grow- 
ing blood vessels has been documented in vivo in a number of physiological, reactive, and pathologic conditions. Newly formed microvessels and/or surrounding stromal cells have been shown to overexpress MMP-1, MMP-2, MMP-3, and MMP-9 (Galis et al, 1994; Karelina et al, 1995; Stricklin et al, 1993; Vacca et al, 1997). In vivo gene ablation experiments have provided further evidence for the angiogenesispromoting effect of MMPs. Knock-out mice deficient for MMP-2 display reduced tumor angiogenesis and tumor progression (Itoh et al, 1998). MMP-9 knock-out mice exhibit defective maturation of the growth plate, probably due to a failure of angiogenesis and enchondral ossification (Vu et al, 1998).

Recent studies have shown that MMPs are overexpressed not only during angiogenesis, but also during the involution and/or breakdown of vascularized organs such as the corpus luteum, the endometrium, and the mammary gland (Ambili et al, 1998; Duncan et al, 1998; Salamonsen, 1994). These findings suggest that proteolytic enzymes may play an important role not only in the initial phases of angiogenesis, but also in the later stages of this process when microvessels no longer proliferate and are gradually reabsorbed. The contribution of enzymatic events to vascular regression is suggested also by the observation that endothelial cells of involuting microvessels round up and detach from the ECM (Modlich et al, 1996). Although these observations implicate MMPs in the reabsorption of microvessels, no studies have been performed to investigate the relationship between MMPs and vascular regression. This gap is largely due to the lack of bioassays capable of reproducing vascular regression under conditions that are suitable for mechanistic studies, quantitative evaluation, and pharmacologic manipulation.

In our laboratory we are using a vascular organ culture model to study wound healing-related angiogenesis and microvessel regression. Explants of rat aorta cultured in collagen gels under serum-free conditions generate outgrowths of microvessels (Nicosia and Ottinetti, 1990). Angiogenesis in this model is a self-limited process mediated by growth factors and other endogenous regulators secreted by aortic cells in response to the injury of the dissection procedure. Because neovessels eventually regress, this model can be used to analyze the molecular mechanisms responsible for the reabsorption of a neovasculature.

In the present study, we describe the relationship between MMP production and microvessel formation and regression in the rat aorta model of angiogenesis. Our results indicate that MMPs promote microvessel formation during the early stages of angiogenesis, but also contribute to the reabsorption of the neovasculature in the later stages of this process. This suggests that MMPs may have either angiogenic or angiolytic effects, depending on the temporal context in which they operate.

\section{Results}

Relationship between Stages of Angiogenesis and MMP Secretion in the Rat Aorta Model

Explants of rat aorta cultured in collagen gel generated microvascular outgrowths in the absence of exogenous growth factors. Microvessels increased progressively in number and then gradually regressed (Nicosia, 1998; Nicosia and Ottinetti, 1990). During the growth phase, microvessels elongated, branched, and formed anastomoses as a result of endothelial cell migration and proliferation. During the regression phase, microvessels retracted and broke down into fragments, which eventually disintegrated. This process was associated with collagen gel lysis around the aortic explants (Fig. 1).

Conditioned media and collagen gel cultures were collected and analyzed by gelatin zymography at different stages of the angiogenic process. The proenzyme and active forms of MMP-2 were detected in conditioned medium and collagen gel, whereas MMP-9 was found predominantly in the gel (Figs. 2 and 3). Western blot analysis confirmed the presence of MMP-2 in conditioned medium and collagen gel, and of MMP-9 in the gel (Fig. 4). Collagen gel extracts

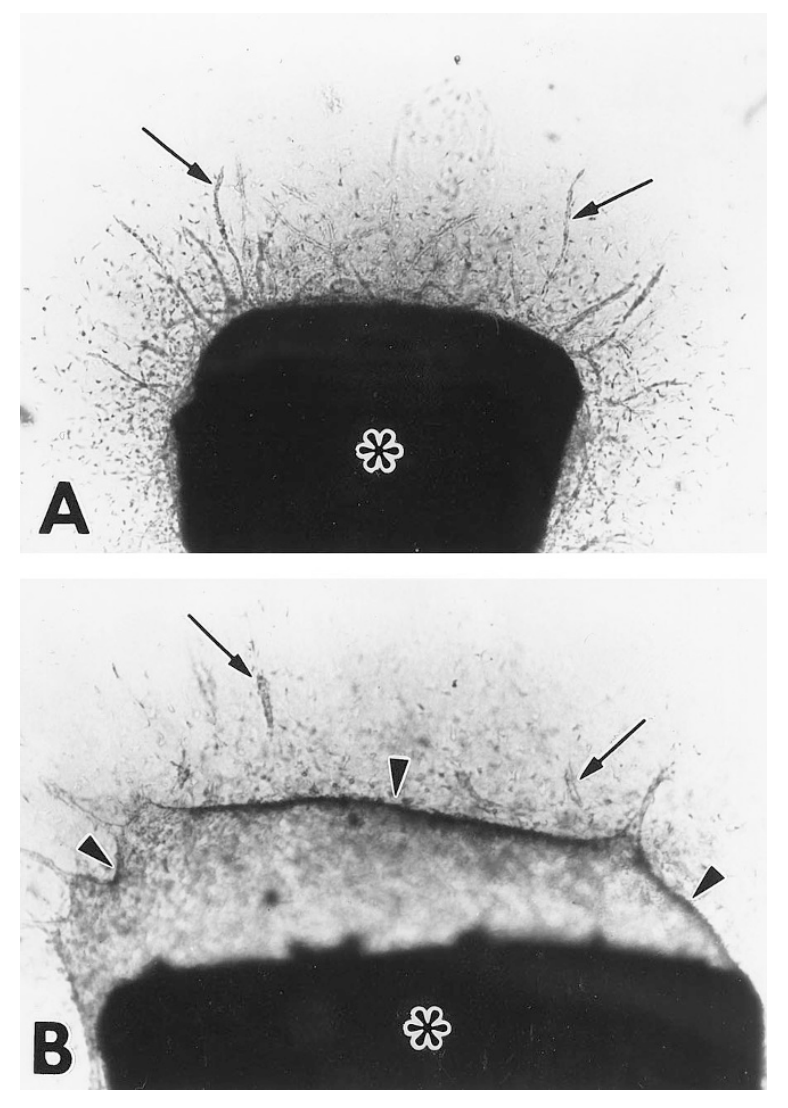

Figure 1.

Serum-free collagen gel cultures of rat aorta photographed during angiogenic (A, Day 7) and vascular regression (B, Day 14) phases. Note that regressing microvessels have become fragmented while the intimal outgrowth from which they have sprouted has lysed the collagen gel (arrowheads). Microvessels are indicated by arrows. The aortic rings are marked by asterisks. Original magnification $\mathrm{A}, \times 35 ; \mathrm{B}, \times 50$. 
DAYS

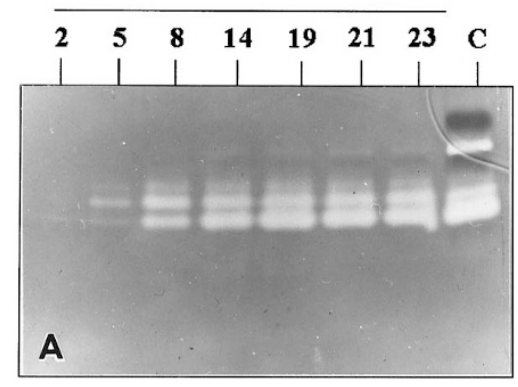

DAYS

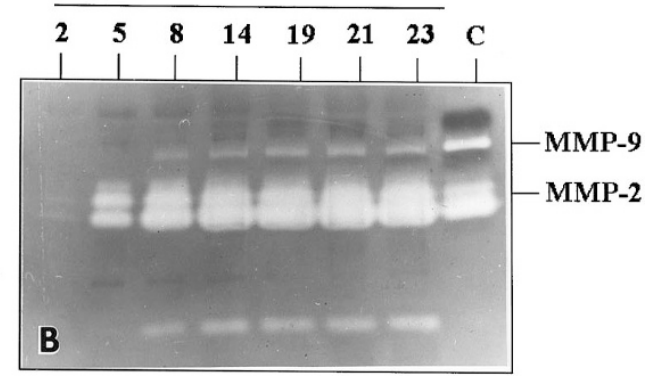

Figure 2.

Gelatin zymograms of $A$, conditioned medium and $B$, collagen gel extracts collected from rat aorta cultures at different time points during formation and regression of microvessels. Note that MMP-2 is found in both culture medium and collagen gel, whereas MMP-9 is predominantly associated with the gel. The collagen gel contains also a lower molecular weight band, which is not visible in the conditioned medium. Expression of MMPs increases during the angiogenic growth phase and remains elevated during the vascular regression phase. C, Gelatinase control: conditioned medium from phorbol myristate acetate-stimulated HT1080 cell line.
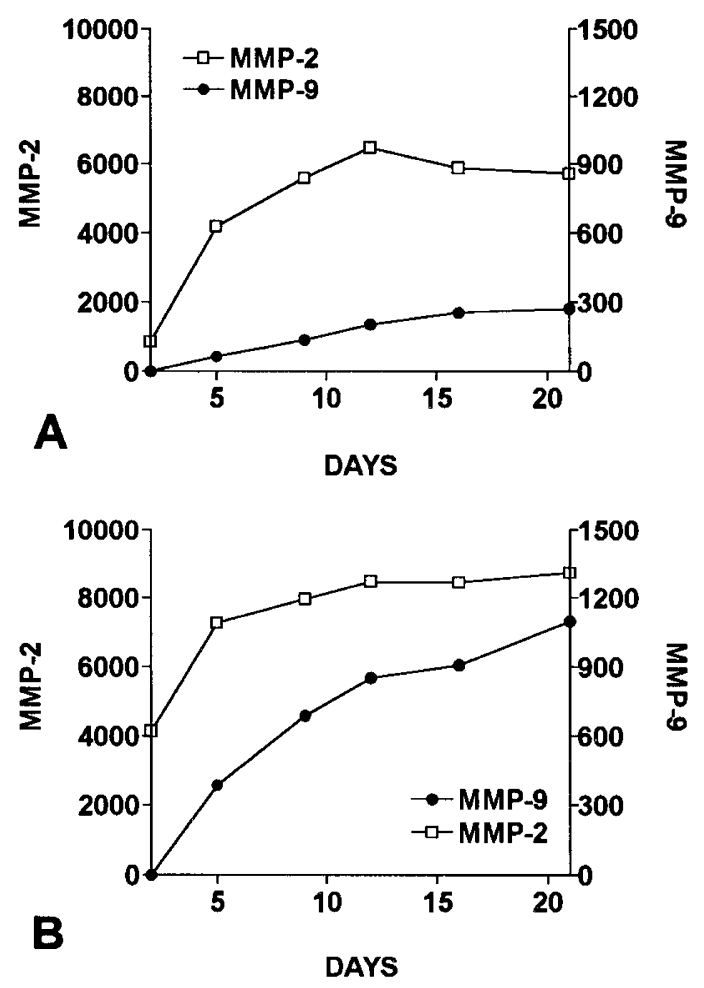

Figure 3 .

Densitometric analysis of MMP-2- and MMP-9-related gelatinase activity in A, conditioned medium and $\mathrm{B}$, collagen gel extracts of rat aorta cultures examined at different stages of angiogenesis (zymograms of Fig. 2). Each data point represents the density of one band. MMP-2 values are obtained by combining the density of proenzyme and active bands. Note that MMP-2 activity reaches its maximum around Day 10 and stays elevated for the remainder of the experiment. MMP-9 activity, which is stronger in the gel, increases gradually over time and is highest at Day 21 . This trend was reproducibly demonstrated in separate experiments.

contained also a gelatinolytic band of low molecular weight (34 kDa, Fig. 2), which did not react with anti-MMP-2 or -MMP-9 antibodies on Western blot analysis. Densitometric evaluation of zymographic bands showed that MMP-2 activity increased gradually in both conditioned medium and gel during the angiogenic growth phase, reached peak levels around Day 10, and remained elevated through the vascular

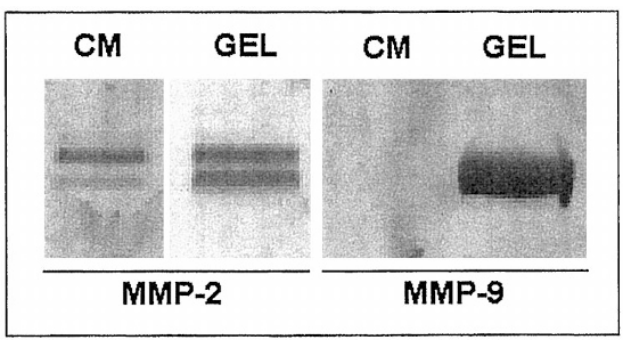

Figure 4.

Western blot of conditioned medium and collagen gel extracts of rat aorta (21-day-old culture) immunostained for MMP-2 and MMP-9. Note that MMP-2 (proenzyme and lower molecular weight active form) is present in both conditioned medium (CM) and gel, whereas MMP-9 is demonstrated only in the gel.

regression phase (Fig. 3). MMP-9 activity continued to increase during the second and third week of culture, reaching its highest level at Day 21, when more than $80 \%$ of the neovessels had regressed (Fig. 3). MMP-2 and MMP-9 levels were $40 \%$ and $315 \%$ higher in the gel than in the conditioned medium, respectively.

\section{Effect of MMP Inhibitors on Rat Aortic Angiogenesis}

The synthetic MMP inhibitors batimastat and marimastat were used to evaluate the functional role of MMPs in the angiogenic response of rat aortic rings. These inhibitors block the enzyme activity of MMPs by binding the zinc ion in the active site of the MMPs (Rasmussen and McCann, 1997). Batimastat and marimastat dose-dependently inhibited microvessel growth during the first week of culture (Fig. 5). This effect was associated with a marked reduction in periaortic collagen lysis (Fig. 6). Although reduced in number, microvessels formed in the presence of batimastat or marimastat survived longer than those of untreated controls. As a result, toward the end of the experiment, MMP inhibitor-treated cultures had a higher number of microvessels compared with the untreated controls in which microvessels were regressing at a fast rate. The angiogenic growth curve of MMP-inhibitor-treated cultures was delayed with a sustained plateau, whereas that of untreated controls exhibited a steep slope after the growth phase. To 

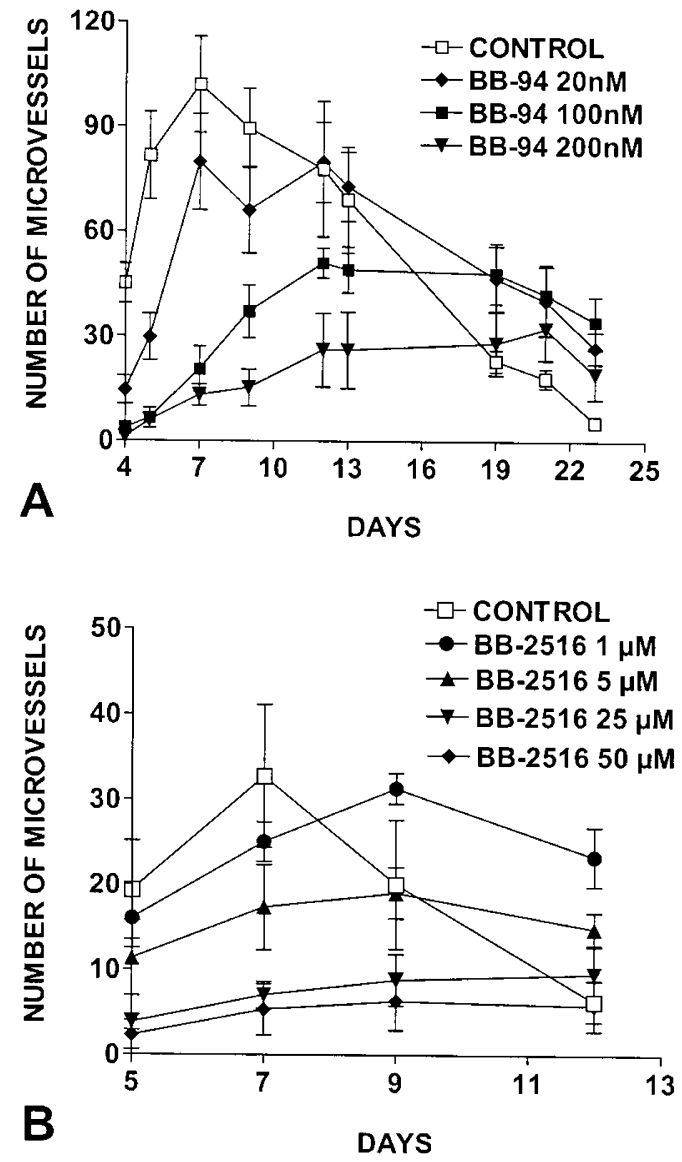

Figure 5.

Dose- and time-dependent effect of the MMP inhibitors A, batimastat (BB-94) and $\mathrm{B}$, marimastat (BB-2516) on microvessel growth and stabilization in serum-free collagen gel culture of rat aorta. Note that batimastat and marimastat inhibit angiogenesis during the first week of growth (Day 7: 100 and $200 \mathrm{nM} \mathrm{BB}-94, p<0.001 ; 25$ and $50 \mu \mathrm{M} \mathrm{BB}-2516, p<0.01$ ). The microvessels formed in the presence of MMP inhibitors are more stable than microvessels of control cultures, which tend to regress. At the end of the experiments (Day 23 for BB-94 and Day 12 for BB-2516) the number of microvessels is significantly higher in cultures treated with batimastat (20 and $100 \mathrm{nM}, p<0.05)$ and marimastat $(1 \mu \mathrm{M}, p<0.01) . n=4$; error bars $=$ SEM.

further understand the effect of MMP inhibitors on vascular regression, batimastat or marimastat was added to rat aortic cultures after the growth phase. Both compounds significantly inhibited microvessel regression (Fig. 7), as well as collagen lysis.

\section{Effect of MMP Inhibitors on bFGF-stimulated Cultures}

Although aortic rings give rise to neovessels spontaneously, they can produce a much greater response if stimulated with exogenous angiogenic factors (Nicosia et al, 1994b). This ex vivo approach mimics in vivo physiologic or pathologic conditions that are characterized by high levels of angiogenic factors and prominent angiogenesis. On this basis, bFGF-treated cultures of rat aortic rings were used to evaluate the effect of MMP inhibitors under conditions of enhanced angiogenic stimulation (Villaschi and Nicosia, 1993). Addition of $12.5 \mathrm{ng} / \mathrm{ml}$ bFGF induced a $320 \%$ stimulation of microvessel number and a $240 \%$ stimulation

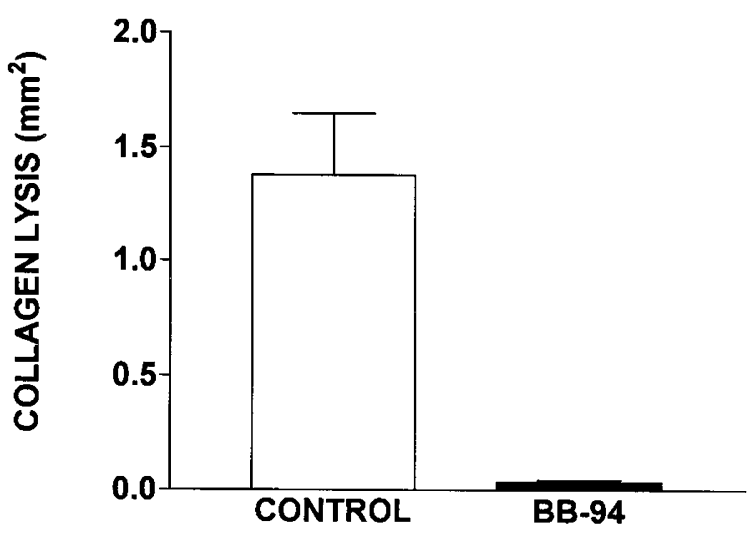

Figure 6.

Morphometric evaluation of batimastat (BB-94) effect on periaortic collagen lysis. Note that the halo of periaortic lysis typically observed in rat aorta cultures during vascular regression (see Fig. 1) is abolished by treatment with $200 \mathrm{nM}$ batimastat. $n=7$ (control), $n=8$ (BB-94); error bars $=$ SEM.
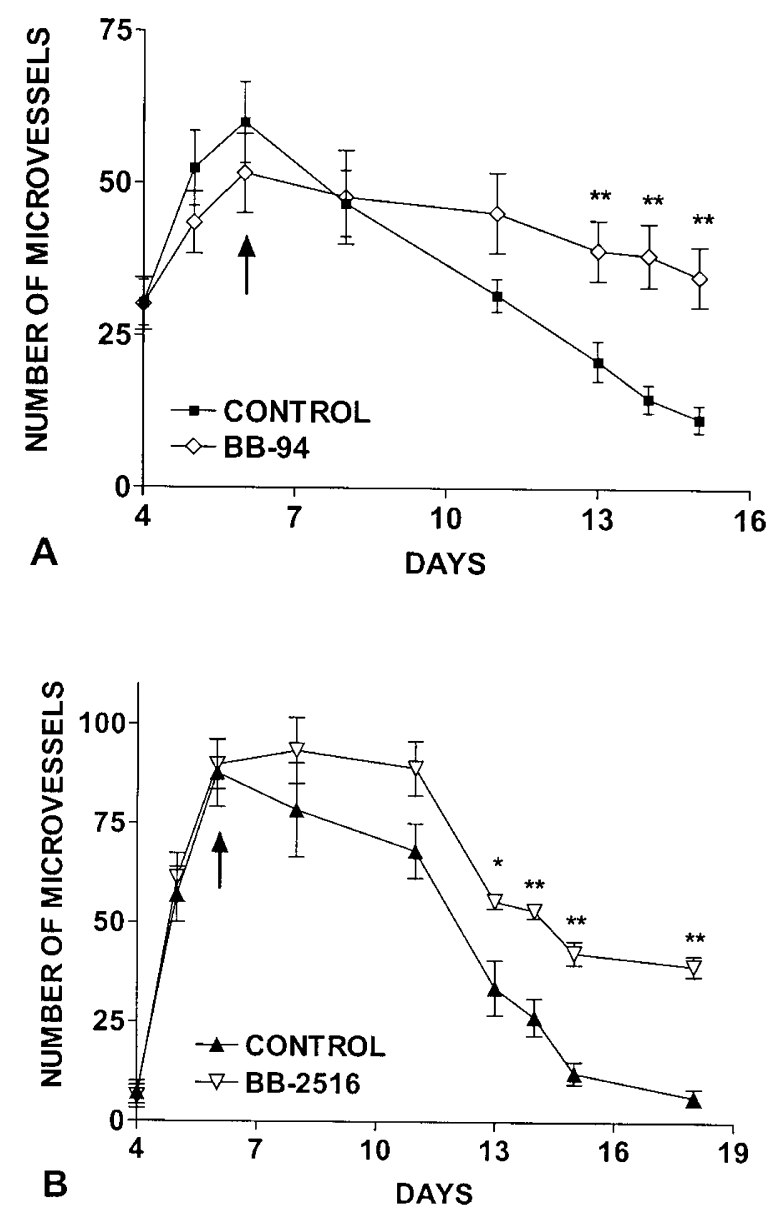

Figure 7.

Collagen gel cultures of rat aorta treated with A, $200 \mathrm{nM}$ batimastat (BB-94, $n=$ 8) or B, $500 \mathrm{nM}$ marimastat (BB-2516, $n=4$ ) after the angiogenic growth phase have more microvessels than the untreated controls because of a markedly reduced rate of vascular regression $\left({ }^{\star} p<0.05 ;{ }^{* \star} p<0.01\right)$. Error bars $=$ SEM.

of microvessel length over control values. Microvessels in bFGF-stimulated cultures proliferated until Day 9 and then gradually regressed. In the presence of marimastat, bFGF-stimulated growth of microvessels 
was significantly delayed. Microvessels in these cultures were also much shorter than those formed in the absence of marimastat (Fig. 8). Microvessel number in marimastat-treated cultures, however, increased steadily and reached a plateau, showing no sign of regression, whereas extensive microvessel regression was observed in bFGF-stimulated cultures not treated with marimastat (Fig. 9). Vascular stabilization in marimastat-treated cultures was associated with markedly reduced collagen lysis. These results demonstrate that neovessels can be stabilized by an MMP inhibitor even under conditions of enhanced angiogenic stimulation by an exogenous growth factor. Taken together, experiments with the rat aorta model indicate that although MMP inhibitors block microvessel sprouting in the early stage of angiogenesis, they also prevent microvessel regression and have the capacity to promote vascular survival.

\section{Stabilization of Endothelial Networks by MMP Inhibitor}

Microvascular networks obtained by culturing rat aortic endothelial cells between two layers of collagen disintegrated within 3-4 days (Villaschi and Nicosia, 1994). Addition of the MMP inhibitor batimastat to the growth medium stabilized the endothelial networks (Fig. 10). After 12 days, digitizing morphometry demonstrated a $300 \%$ greater microvessel survival in cultures treated with $200 \mathrm{~nm}$ batimastat over untreated controls. This observation supports the idea that vascular regression in collagen gels is mediated by MMPs.

\section{Accumulation of Subendothelial Collagen in Microvessels Stabilized by MMP Inhibitor}

Ultrastructural evaluation of bFGF-induced microvessels stabilized by the MMP inhibitor marimastat demonstrated an abundant subendothelial matrix rich in collagen (Fig. 11A). The abluminal surface of the marimastat-treated endothelium was flat and tightly adherent to the underlying collagen fibrils (Fig. 11B). Endothelial cells were spread out and well differentiated. bFGF-treated microvessels without marimastat

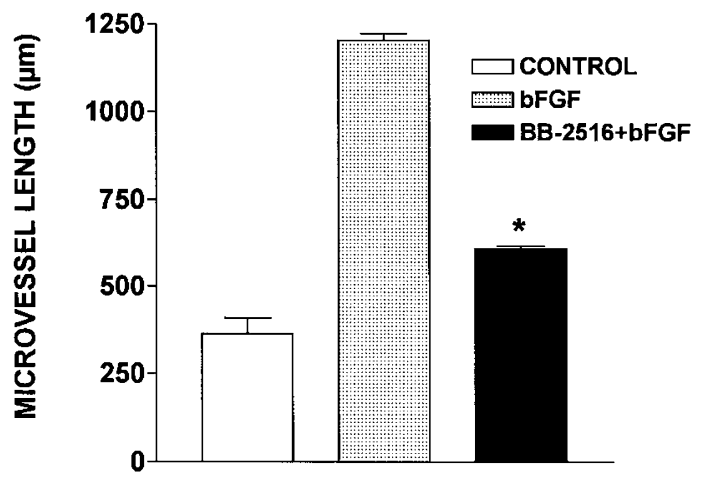

Figure 8.

Effect of bFGF on length of microvessels in the presence or absence of marimastat (BB-2516). Note that marimastat $(0.5 \mu \mathrm{M})$ markedly reduced bFGF $(12.5 \mathrm{ng} / \mathrm{ml})$-induced elongation of microvessels ( $\left.{ }^{\star} p<0.001\right) . n=60$. Error bars $=$ SEM.

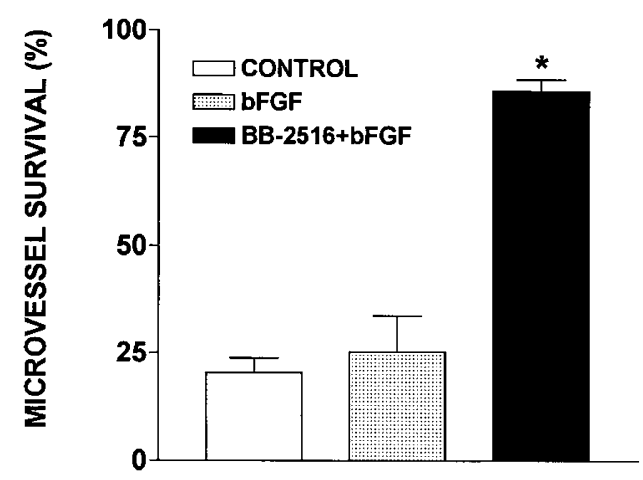

Figure 9.

Effect of marimastat on microvessel survival in bFGF-stimulated cultures of rat aorta. Microvessel survival, expressed as the percentage of microvessels that survived at Day 16, is calculated with the following formula: $\mathrm{N}^{16} / \mathrm{N}^{\mathrm{M}} \times 100$, where $\mathrm{N}^{16}$ is the number of microvessels at Day 16 , and $\mathrm{N}^{\mathrm{M}}$ is the maximum number of microvessels formed in each culture. Microvessels were significantly more stable $\left({ }^{*} p<0.001\right)$ in marimastat-treated cultures than in bFGF-treated cultures or untreated controls. $n=4$; error bars $=$ SEM.
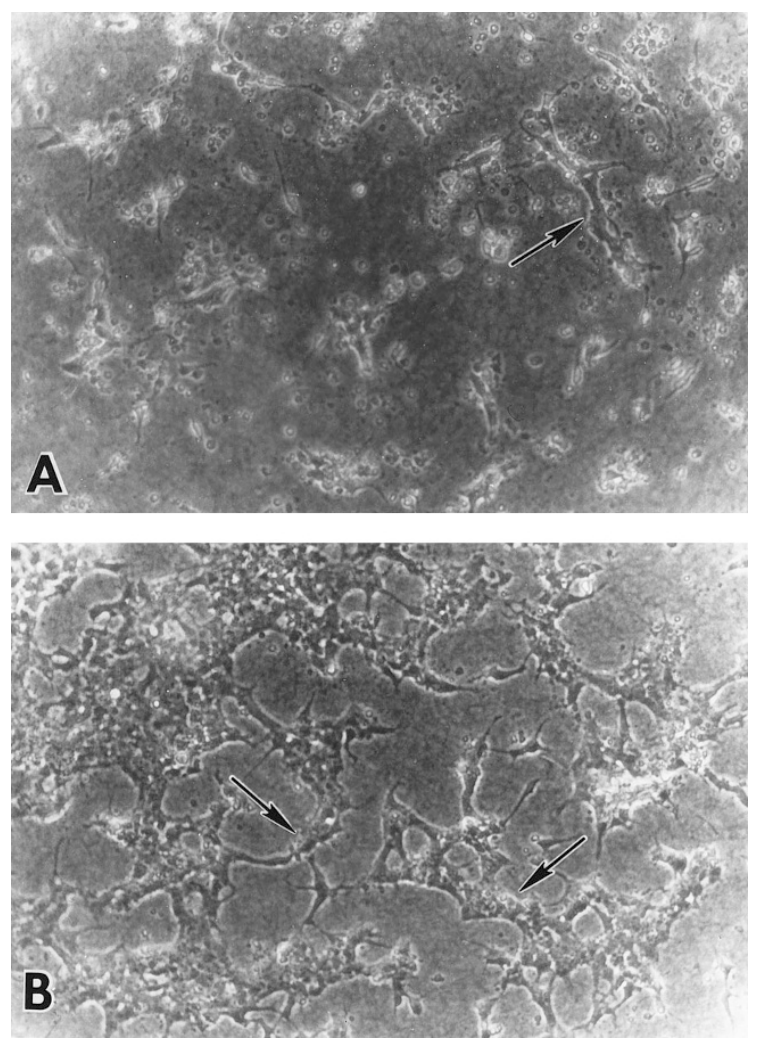

Figure 10.

Effect of batimastat on microvascular networks in 12-day-old serum-free collagen gel overlay culture of rat aortic endothelial cells. Note that microvascular networks (arrows) formed by endothelial cells disintegrated $\mathrm{A}$, in the untreated control and B, were stabilized by $200 \mathrm{nM}$ batimastat. The cultures were maintained in BB-94-containing medium from the beginning of the experiment. Original magnification, $\times 50$.

were surrounded by a halo of collagen lysis (Fig. 12A). Collagen breakdown resulted in endothelial cell detachment from the ECM and was accompanied by the formation of many abluminal blebs and vesicles (Fig. 12B). Endothelial cells disengaged from the surrounding collagen became rounded and contracted. 

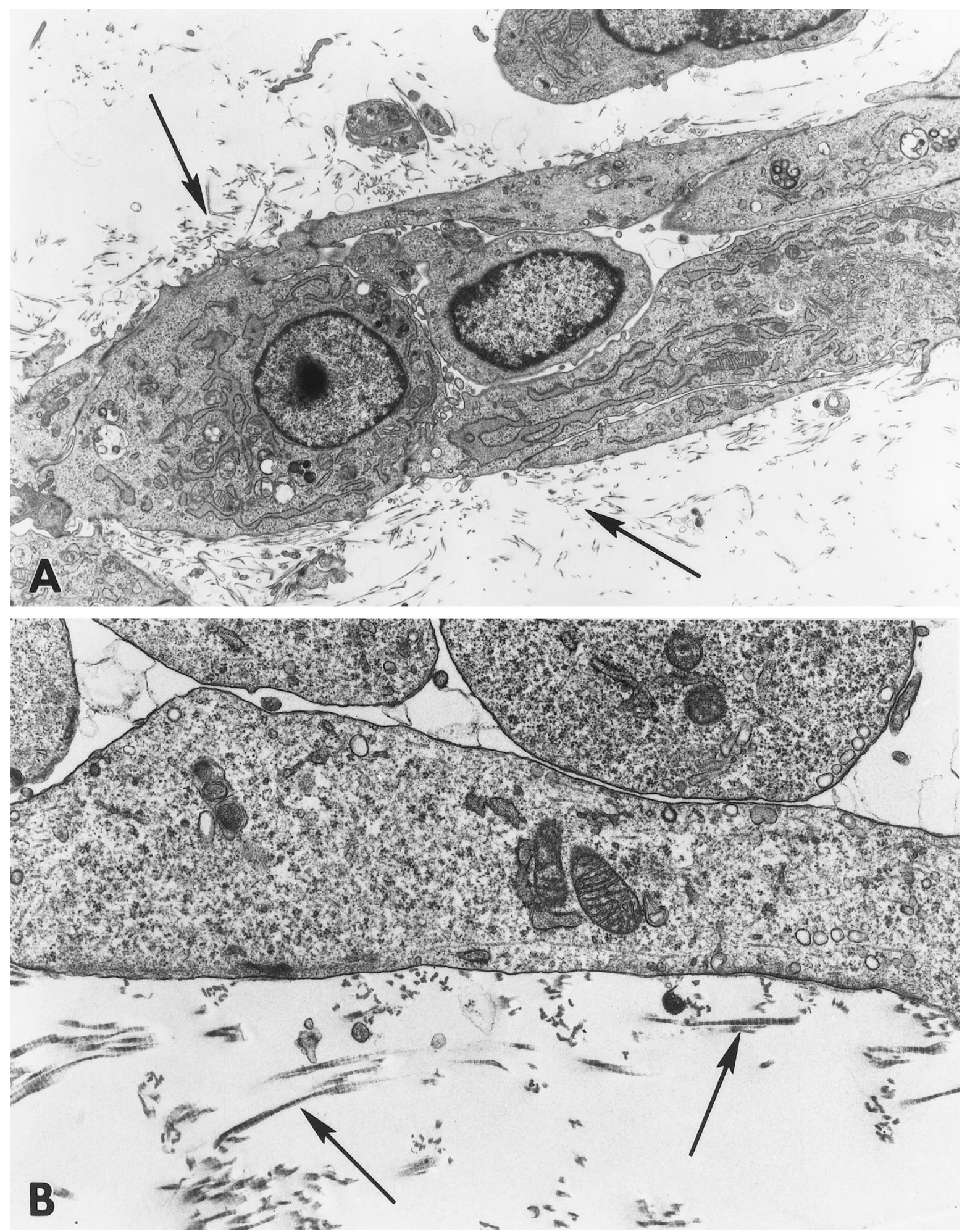

\section{Figure 11.}

Electron micrograph of bFGF-induced microvessel in collagen gel culture of rat aorta treated with marimastat $(1 \mu \mathrm{M})$. Note that the microvessel is surrounded by abundant collagen fibrils (A, B: arrows). The endothelium is tightly anchored to the underlying collagen and has a flat and compact abluminal surface. Original magnification, $A, \times 12,000 ; B, \times 37,000$.

\section{Discussion}

The angiogenic response of the rat aorta is a selflimited process characterized by a growth phase followed by a regression phase during which neovessels are gradually reabsorbed. In this paper we report that the reabsorbtion of the aorta-derived neovascu- 

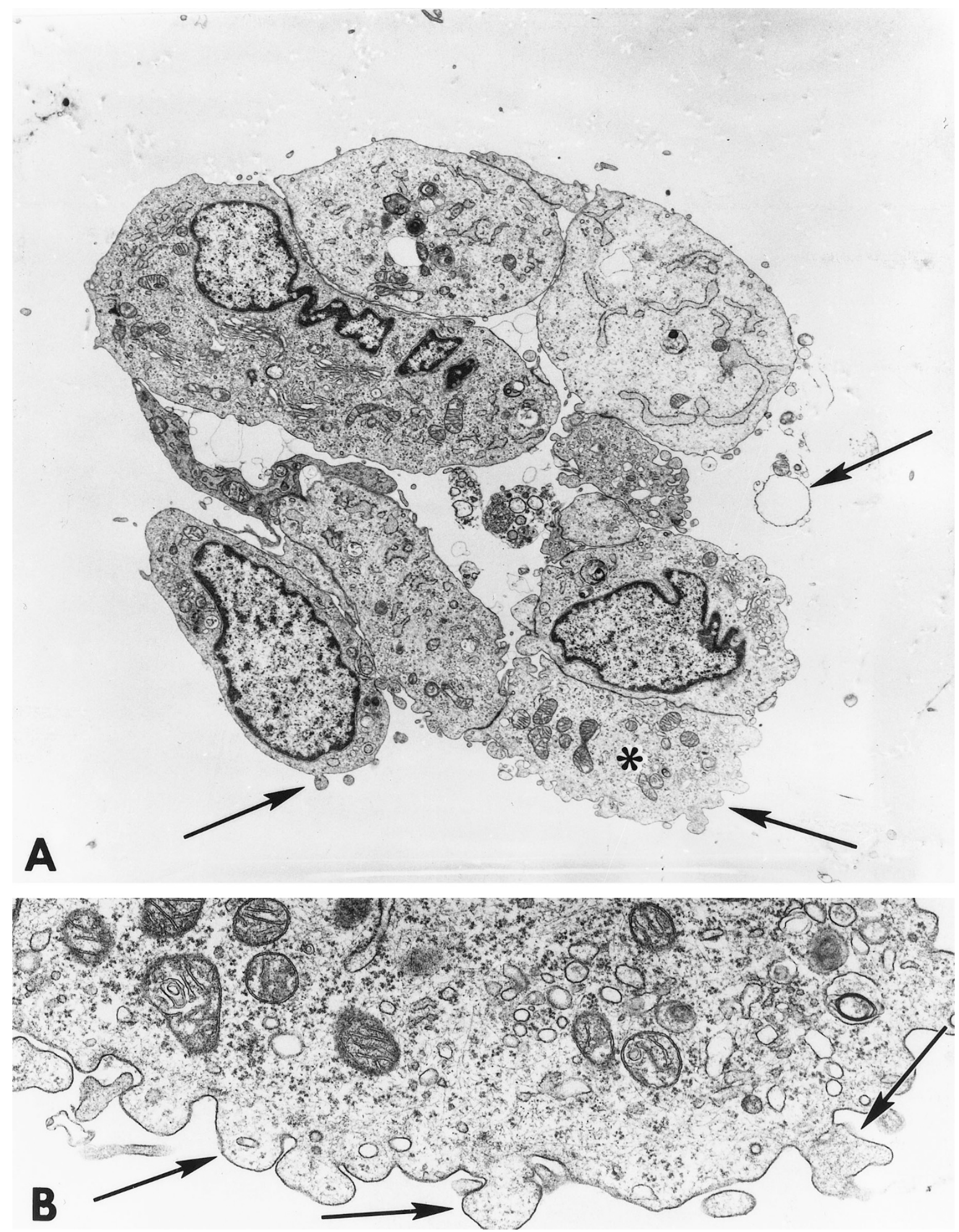

\section{Figure 12}

Electron micrograph of bFGF-induced microvessel in collagen gel culture of rat aorta during vascular regression phase. A, Note that the microvessel has detached from the underlying interstitial collagen. B, A step-section of the area marked by an asterisk is shown enlarged in the bottom panel. Detached endothelial cells exhibit many abluminal blebs and vesicles (arrows). Original magnification, A, $\times 12,000 ; \mathrm{B}, \times 37,000$.

lature is regulated by MMPs. This interpretation is based on the following observations: (1) angiogenesis in the rat aorta model is associated with secretion of MMP-2 and MMP-9 by the aortic explant and its outgrowth; (2) MMP levels, which increase steadily during angiogenesis, remain elevated during vascular regression; (3) vascular regression correlates with perivascular collagen lysis; (4) synthetic MMP inhibi- 
tors not only block rat aortic angiogenesis and collagen lysis, but also stabilize the aorta-derived microvessels and prevent vascular regression; (5) inhibition of MMPs results in the survival of microvascular networks formed by isolated endothelial cells in serum-free collagen gel culture. Based on these observations, we propose that MMPs have a timedependent dual function in angiogenesis. They promote the formation of microvessels, but also contribute to the reabsorption and regression of the neovasculature.

The ex vivo angiogenic response of the rat aorta is akin to the in vivo neovascular responses of injured tissues during wound healing and represents a model of physiologic/reactive angiogenesis. The morphologic changes of regressing microvessels in rat aorta cultures are similar to changes that have been described during vascular regression in vivo. In the rat aorta model, endothelial cells of regressing microvessels detached from the underlying collagen, which was lysed. In addition, regressing microvessels retracted and broke down into fragments. Detachment of endothelial cells from the basement membrane has been observed in the vasculature of the corpus luteum during its involution (Modlich et al, 1996). A similar process was reported by Clark and Clark (1939), who noticed that vessels formed during wound healing in the transparent rabbit ear chamber regressed by endothelial retraction if they failed to anastomose with other vessels and were not perfused with blood. The observation that vascular regression is associated with endothelial cell rounding and detachment from the underlying matrix supports the idea that this process is due to unbalanced pericellular proteolysis, which disrupts endothelial cell adhesion to the ECM. The formation of numerous cytoplasmic blebs at the abluminal surface of the endothelium of regressing vessels in the rat aorta model is consistent with this interpretation, because cytoplasmic blebbing is typically seen in cells that are detached from culture substrates by proteolytic enzymes (Bereiter-Hahn et al, 1990). Thus, continuous MMP activity after vessels have formed results in progressive loss of the ECM scaffold that is critical for vascular integrity. Without an adequate substrate, endothelial cells, which are anchorage-dependent (Boudreau et al, 1996; Stromblad and Cheresh, 1996), fail to survive and the neovessels are gradually reabsorbed.

Evidence for a role of MMPs in the proteolytic events of vascular regression can be found in studies that describe the temporal expression of these enzymes in tissues with active angiogenic responses. For example, MMP-9 activity peaks in both early and late luteal phases and MMP-2 activity is maximal in the late corpus luteum, when vessels are regressing (Duncan et al, 1998). A similar observation was made in the endometrium, in which MMPs are overexpressed during the late menstrual phase, when hemorrhage occurs as a result of vessel breakdown (Salamonsen, 1994). MMP up-regulation has been implicated also in the involution of the mammary gland (Ambili et al, 1998).
We previously reported that aorta-derived microvessels survived longer if cultured in collagen gels supplemented with high doses of basement membrane molecules (Nicosia et al, 1994a). We also found that disruption of endothelial cell-ECM interactions with RGD-containing peptides caused disintegration of neovessels (Nicosia and Bonanno, 1991; Nicosia et al, 1993a). The results of the present study are consistent with these observations, because they indicate that preservation of the ECM scaffold around the endothelium through inhibition of MMP activity results in prolonged vessel survival.

Our observation that synthetic MMP inhibitors dose-dependently block the angiogenic response of the rat aorta indicates that these enzymes play also an important role in promoting microvessel formation during the early stages of angiogenesis. This is consistent with reports by other laboratories that have emphasized the importance of ECM proteolysis in the initiation of angiogenesis. MMPs, which have been localized by in situ labeling techniques in developing microvessels (Karelina et al, 1995; Stricklin et al, 1993; Vacca et al, 1997) are believed to be necessary for angiogenesis because neutralization of MMP activity inhibits formation of capillaries from isolated endothelial cells (Haas et al, 1998; Qian et al, 1997; Schnaper et al, 1993). In addition, MMP-2 knock-out mice display reduced tumor angiogenesis (Itoh et al, 1998), and MMP-9 knock-out mice have been reported to have defective maturation of the growth plate, probably due to a failure of angiogenesis (Vu et al, 1998).

It is possible that MMPs promote angiogenesis in the growth factor-rich milieu that surrounds the endothelium of immature microvessels and vascular regression in the growth factor deficient environment that prevails in the late stages of this process. This interpretation would explain why neovessels regress during physiologic/reactive processes, which are characterized by a gradual depletion of growth factors (Nicosia et al, 1997; Villaschi and Nicosia, 1993), but not in pathologic processes such as cancer, in which growth factors levels are constantly elevated (Hazelton et al, 1999; Li et al, 1994).

In the rat aorta model, MMP production during the angiogenic growth phase is probably stimulated by angiogenic factors. For example, bFGF, which is released by the aortic explants after injury (Villaschi and Nicosia, 1993), has the capacity to induce MMP production by isolated endothelial cells (Cornelius et al, 1995) and rat aortic explants (unpublished observations, Zhu and Nicosia). Persistent MMP production and/or activation in the absence of growth factor stimuli during the vascular regression phase may be due to cell-cell and/or cell-matrix interactions. For example, MMP-2 synthesis is modulated by interstitial collagen and intracellular calcium influx (Haas et al, 1998; Ray and Stetler-Stevenson, 1994), whereas MMP-9 can be induced by thrombospondin-1 (Qian et al, 1997). In addition, MMP-2 can be activated by cell-associated MMPs such as membrane-type-1 metalloproteinase (Haas et al, 1998; Sato et al, 1994). 
The stabilizing effect on aorta-derived microvessels of synthetic MMP inhibitors such as batimastat and marimastat raises the possibility that endogenous MMP inhibitors such as TIMPs may play a role in the mechanisms of vascular survival. TIMPs are known to have anti-angiogenic effects (Murphy et al 1993; Schnaper et al, 1993), but their capacity to inhibit vascular regression through blockage of MMP function has not been investigated. Future studies should explore the effect of changes in the MMP/TIMP balance on the fate of neovascular outgrowths in physiologic, reactive and pathologic conditions. It will also be important to investigate the role of specific MMPs at different stages of microvessel development. The observation that MMP-2 and MMP-9 have different patterns of distribution in the solid and soluble phases of rat aorta cultures raises the intriguing possibility that these molecules may have distinct roles in the angiogenic process. Of particular interest is MMP-9, which tends to accumulate in the solid phase and whose expression correlates with both angiogenesis and the reabsorption of neovessels.

Understanding the mechanisms by which MMPs regulate vascular regression may have important clinical implications because the majority of patients with angiogenesis-dependent disorders become symptomatic when angiogenesis has already occurred. Antiangiogenic therapy with drugs that block formation of microvessels should prevent progression of the disease process, but a therapy designed to induce vascular regression may ultimately be needed for the curative treatment of preexisting lesions. To that end, the hypothesis that perivascular proteolysis is a mechanism of neovessel reabsorption warrants further investigation.

\section{Materials and Methods}

\section{Rat Aorta Model of Angiogenesis}

Aortic rings obtained from 5- to 10-week-old Fischer 344 male rats were embedded in collagen gels and transferred to 16-mm wells (4-well NUNC dishes) each containing $0.5 \mathrm{ml}$ serum-free endothelial basal medium (EBM, Clonetics Corporation, San Diego, California) (Nicosia, 1998). The growth medium was changed 3 times a week starting from Day 3 . The cultures were treated with increasing concentrations of the MMP inhibitors batimastat or marimastat (British Biotech Pharmaceutical Limited, Oxford, UK) in the presence or absence of bFGF (R and D Systems, Minneapolis, Minnesota). Controls included cultures treated with vehicle alone (dimethyl sulfoxide) and untreated cultures. MMP inhibitors were added to the medium from the beginning of the experiment or at Days 6 to 7, toward the end of the angiogenic phase, and supplemented with each feeding.

\section{Collagen Gel Overlay Assay of Microvascular Tube Formation}

Rat aortic endothelial cells isolated from tissue explants were seeded on collagen gels under serum-free conditions, allowed to attach and spread overnight, and overlaid with a second layer of collagen, as described (Nicosia et al, 1993b). Endothelial cells cultured in this manner reorganized into networks of microvascular tubes within 24 hours. Triplicate collagen gel overlay cultures were treated with the MMP inhibitor batimastat (200 nM) or left untreated.

\section{Electron Microscopy}

For transmission electron microscopy, collagen gel cultures of rat aorta were fixed in 3\% glutaraldehyde, $0.1 \mathrm{M}$ cacodylate buffer, $\mathrm{pH}$ 7.4. They were then postfixed in osmium tetroxide, en bloc stained with $2 \%$ aqueous uranyl acetate, embedded in EPON, thin sectioned, stained with lead citrate, and examined with a Zeiss $10 \mathrm{~A}$ transmission electron microscope.

\section{Measurement of Angiogenesis}

The angiogenic response of aortic cultures was determined by counting the number of microvessels in the living cultures, according to published criteria (Nicosia and Ottinetti, 1990). The elongation of microvessels and the area of collagen lysis surrounding the aortic rings were measured by digitizing morphometry using Bioquant IV image analysis software (Nicosia et al, 1993). Microvascular elongation was quantified by measuring the shortest distance between the tip of the vessel to the edge of the aorta ring. Each experimental group included the 60 longest microvessels of triplicate cultures.

For the collagen gel overlay assay, cultures were measured by digitizing morphometry. Collagen gels were fixed in $10 \%$ buffered formalin, washed in distilled water, transferred to a histology glass slide, and air dried to obtain whole mounts. Each gel was rehydrated with distilled water before quantitative evaluation. Microvessels were visualized under bright field microscopy at $\times 4$ magnification. Each culture was scored by measuring the cumulative length/field of five randomly selected microvascular networks.

\section{Statistical Analysis}

Experiments with aortic cultures included three to four cultures per group and were repeated at least three times. Collagen gel overlay experiments with isolated endothelial cells were repeated twice. Data were analyzed with GraphPad Prism statistics software (GraphPad Software Inc., San Diego, California). Student's $t$ test or one way ANOVA followed by NewmanKeuls multiple comparison test was used to evaluate whether differences among groups were significant. Statistical significance was set at $p<0.05$.

\section{Gelatin Zymography}

Conditioned media or collagen gels each housing the aortic explant and its outgrowth were mixed with the appropriate volume of $5 \times$ sodium dodecyl sulfate (SDS) sample buffer to obtain a final $1 \times$ buffer concentration $(0.08 \mathrm{M}$ Tris- $\mathrm{HCl} \mathrm{pH} 6.8,1 \%$ SDS, 4\% 
glycerol, $0.006 \%$ bromophenol blue). The samples were kept at room temperature for 20 minutes and subjected to electrophoresis in $8.5 \%$ SDS-PAGE containing $0.2 \%$ gelatin at $4^{\circ} \mathrm{C}$ (Kleiner and StetlerStevenson, 1994). The electrophoresis gels were washed in $2.5 \%$ Triton $\mathrm{X}-100$ to remove SDS and developed for 24 hours at $37^{\circ} \mathrm{C}$ in $50 \mathrm{~mm}$ Tris- $\mathrm{HCl}$, $\mathrm{pH}$ 7.4, $0.15 \mathrm{M} \mathrm{NaCl}, 10 \mathrm{~mm} \mathrm{CaCl}_{2}$ and $0.02 \% \mathrm{NaN}_{3}$. They were then stained with $0.1 \%$ Coomassie brilliant blue R250 solution in 10\% acetic acid, washed with $30 \%$ isopropanol in 10\% acetic acid, and dried. The intensity of the gelatinolytic bands corresponding to MMP-2 and MMP-9 was measured with a Personal Densitometer SI (Molecular Dynamics, Sunnyvale, California).

\section{Western Analysis}

Conditioned media and collagen gels from rat aorta cultures were subjected to SDS-PAGE, transferred to nitrocellulose membrane, immunostained with a rabbit polyclonal antibody against MMP-2 kindly provided by Dr. W.G. Stetler-Stevenson (National Cancer Institute, Bethesda, Maryland) or a monoclonal mouse antibody against MMP-9 (Oncogene, Cambridge, Massachusetts), and reacted with biotinylated secondary antibodies against IgG of the appropriate animal species. Immunoreactive bands were reacted with the ABC kit and visualized with a peroxidase substrate (Vector Laboratories, Inc., Burlingame, California).

\section{References}

Ambili M, Jayasree K, and Sudhakaran PR (1998). 60K gelatinase involved in mammary gland involution is regulated by $\beta$-oestradiol. Biochim Biophys Acta 1403:219-231.

Ausprunk DH and Folkman J (1977). Migration and proliferation of endothelial cells in preformed and newly formed blood vessels during tumor angiogenesis. Microvasc Res 14:53-65.

Bereiter-Hahn J, Luck M, Miebach T, Stelzer HK, and Voth M (1990). Spreading of trypsinized cells: Cytoskeletal dynamics and energy requirements. J Cell Sci 96:171-188.

Boudreau N, Werb Z, and Bissell MJ (1996). Suppression of apoptosis by basement membrane requires threedimensional tissue organization and withdrawal from the cell cycle. Proc Natl Acad Sci USA 93:3509-3513.

Clark ER and Clark EL (1939). Microscopic observation on the growth of blood capillaries in the living mammal. Am J Anat 64:251-299.

Cornelius LA, Nehring LC, Roby JD, Parks WC, and Welgus HG (1995). Human dermal microvascular endothelial cells produce matrix metalloproteinases in response to angiogenic factors and migration. J Invest Dermatol 105:170-176.

Duncan WC, McNeilly AS, and Illingworth PJ (1998). The effect of luteal "rescue" on the expression and localization of matrix metalloproteinases and their tissue inhibitors in the human corpus luteum. J Clin Endocrinol Metab 83:24702478.

Folkman J (1995). Angiogenesis in cancer, vascular, rheumatoid and other diseases. Nat Med 1:27-31.
Folkman $\mathrm{J}$ and Klagsbrun M (1987). Angiogenic factors. Science 235:442-447.

Galis Zs, Sukhova GK, Lark MW, and Libby P (1994). Increased expression of matrix metalloproteinases and matrix degrading activity in vulnerable regions of human atherosclerotic plaques. J Clin Invest 94:2493-2503.

Haas TL, Davis SJ, and Madri JA (1998). Three-dimensional type I collagen latices induce coordinate expression of matrix metalloproteinases MT1-MMP and MMP2 in microvascular endothelial cells. J Biol Chem 273:3604-3610.

Hazelton D, Nicosia RF, and Nicosia SV (1999). Vascular endothelial growth factor levels in ovarian cyst fluid correlate with malignancy. Clin Cancer Res 5:823-829.

Itoh T, Tanioka M, Yoshida H, Yoshioka T, Nishimoto H, and Itohara S (1998). Reduced angiogenesis and tumor progression in gelatinase A-deficient mice. Cancer Res 58:10481051.

Karelina TV, Goldberg GI, and Elsen AZ (1995). Matrix metalloproteinases in blood vessel development in human fetal skin and in cutaneous tumors. J Invest Dermatol 105: 411-417.

Kleiner DE and Stetler-Stevenson WG (1994). Quantitative zymography: Detection of picogram quantities of gelatinases. Anal Biochem 218:325-329.

Li VW, Folkerth RD, Watanabe H, Yu C, Rupnick M, Barnes P, Scott RM, Black PM, Sallan SE, and Folkman J (1994). Microvessel count and cerebrospinal fluid basic fibroblast growth factor in children with brain tumours. Lancet 344: 82-6.

Modlich U, Kaup FJ, and Augustin HG (1996). Cyclic angiogenesis and blood vessel regression in the ovary: Blood vessel regression during luteolysis involves endothelial cell detachment and vessel occlusion. Lab Invest 74:771-780.

Montesano R, Orci L, and Vassalli P (1983). In vitro rapid organization of endothelial cells into capillary-like networks is promoted by collagen matrices. J Cell Biol 97:1648-1652.

Moscatelli D and Rifkin D (1988). Membrane and matrix localization of proteinases: a common theme in tumor cell invasion and angiogenesis. Biochim Biophys Acta 948:6785.

Murphy AN, Unsworth EJ, and Stetler-Stevenson WG (1993). Tissue inhibitor of metalloproteinase-2 inhibits bFGFinduced human microvascular endothelial cell proliferation. J Cell Physiol 157:351-358.

Nicosia RF (1998). The rat aorta model of angiogenesis and its application. In: Mironov V, Little C, and Sage H, editors. Microvascular morphogenesis in vivo, in vitro and in mente. Boston: Birkhauser, 111-139.

Nicosia RF and Bonanno E (1991). Inhibition of angiogenesis in vitro by Arg-Gly-Asp-containing synthetic peptide. Am J Path 138:829-833.

Nicosia RF, Bonanno E, and Smith M (1993a). Fibronectin promotes the elongation of microvessels during angiogenesis in vitro. J Cell Physiol 154:654-661.

Nicosia RF, Bonanno E, and Yurchenco P. (1994a). Modulation of angiogenesis in vitro by laminin-entactin complex. Dev Biol 164:197-206. 
Nicosia RF, Lin YJ, Hazelton D, and Qian X (1997). Endogenous regulation of angiogenesis in the rat aorta model: Role of vascular endothelial growth factor. Am J Path 151:13791386.

Nicosia RF, Nicosia V, and Smith M. (1994b). Vascular endothelial growth factor, platelet derived growth factor, and insulin-like growth factor-1 promote rat aortic angiogenesis in vitro. Am J Pathol 145:1023-1029.

Nicosia RF and Ottinetti A (1990). Growth of microvessels in serum-free matrix culture of rat aorta: A quantitative assay of angiogenesis in vitro. Lab Invest 63:115-122.

Nicosia RF, Villaschi S, and Smith M (1993b). Isolation and characterization of vasoformative endothelial cells from the rat aorta. In vitro Cell Dev Biol 30A:394-399.

Qian X, Wang TN, Rothman VL, Nicosia RF, and Tuszynski GP (1997). Thrombospondin-1 modulates angiogenesis in vitro by up-regulation of matrix metalloproteinase- 9 in endothelial cells. Exp Cell Res 235:403-412.

Rasmussen HS and McCann PP (1997). Matrix metalloproteinase inhibition as a novel anticancer strategy: A review with special focus on batimastat and marimastat. Pharmacol Therap 75:69-75.

Ray JM and Stetler-Stevenson WG (1994). The role of matrix metalloproteases and their inhibitors in tumor invasion, metastasis and angiogenesis. Eur Resp J 7:2062-2072.

Salamonsen LA (1994). Matrix metalloproteinases in endometrial remodelling. Cell Biol Int 18:1139-1145.

Sato H, Takino T, Okada Y, Cao J, Shinagawa A, Yamamoto E, and Seiki M (1994). A matrix metalloproteinase expressed on the surface of invasive tumor cells. Nature 370:61-65.

Schnaper HW, Grant DS, Stetler-Stevenson WG, Fridman R, D'Orazi G, Murphy AN, Bird RE, Hoythya M, Fuerst TR, French DL, Quigley JP, and Kleinman HK (1993). Type IV collagenase(s) and TIMPs modulate endothelial cell morphogenesis in vitro. J Cell Physiol 156:235-246.
Stricklin GP, Li L, Jancic V, Wenczak BA, and Nanney L (1993). Localization of mRNAs representing collagenase and TIMP in sections of healing human burn wounds. Am J Pathol 143:1657-1666.

Stromblad S and Cheresh DA (1996). Integrins, angiogenesis and vascular cell survival. Chem Biol 3:881-885.

Unemori EN, Ferrara N, Bauer EA, and Amento EP (1992). Vascular endothelial growth factor induces interstitial collagenase expression in human endothelial cells. J Cell Physiol 153:557-562.

Vacca A, Moretti S, Ribatti D, Pellegrino A, Pimpinelli N, Bianchi B, Bonifazi E, Ria R, Serio G, and Dammacco F (1997). Progression of mycosis fungoides is associated with changes in angiogenesis and expression of the matrix metalloproteinases 2 and 9. Eur J Cancer 33:1685-1692.

Villaschi S and Nicosia RF (1993). Angiogenic role of basic fibroblast growth factor released by rat aorta after injury. Am J Pathol 143:182-190.

Villaschi S and Nicosia RF (1994). Paracrine interactions between fibroblasts and endothelial cells in a serum-free co-culture model: Modulation of angiogenesis and collagen gel contraction. Lab Invest 71:291-299.

Vu TH, Shipley JM, Bergers G, Berger JE, Helms JA, Hanahan D, Shapiro SD, Senior RM, and Werb Z (1998). MMP-9/ gelatinase $B$ is a key regulator of growth plate angiogenesis and apoptosis of hypertrophic chondrocytes. Cell 93:411422. 